\title{
Centrales hydroélectriques : conséquences économiques et écologiques de variantes d'équipement et d'exploitation
}

\author{
P. Maire (1), J. F. Bansard (2), T. Do (3) \\ (1) Conseil Supérieur de la Pêche-DR de Metz \\ (2) THEE \\ (3) DIREN Lorraine
}

\begin{abstract}
Le renouvellement d'autorisations de centrales hydroélectriques existant avant 1919 conduit à examiner et instruire dans un laps de temps très court un grand nombre de dossiers selon une procédure administrative identique à celle d'une installation nouvelle. Cette simultanéité présente un inconvénient: on ne bénéficie pas ou peu du retour d'expérience que permettraient des prescriptions accordées successivement sur des ouvrages de même nature ; elle présente un avantage: le thème permet une réflexion globale, la mise au point de techniques standard d'évaluation et de prescription. Cette note s'inscrit dans cette réflexion inspirée par l'examen de plus d'une centaine de dossiers de demande d'autorisation de création et principalement de renouvellement. Le département des Vosges est, dans l'Est de la France particulièrement concerné.
\end{abstract}

\section{CIRCONSTANCES}

La structure réglementaire des études ou notice d'impact (décret d'octobre 1977), prévoit l'examen des variantes et l'exposé des raisons du choix. Parmi les variantes et dans l'esprit de la loi de 76 on compte trouver celle qui présente le meilleur rapport, le meilleur compromis «EconomieEcologie ».

L'économie, c'est la production d'un maximum de kW.h par l'exploitation d'un volume maximum d'eau (variable pseudo aléatoire dépendant des débits naturels) sous une chute donnée (composante fixe, pour les moyennes et hautes chutes dont il sera seulement question ici) qui dépend de l'aménagement possible ou existant du site.

L'écologie, c'est compenser et réduire autant que possible les perturbations du milieu naturel liées à l'installation (barrage) et à l'exploitation de l'industrie. La réduction du débit dans le tronçon court-circuité est la perturbation qui sera principalement envisagée ici.

Malgré des progrès significatifs dans les études et présentations des dossiers de demande d'autorisation, il n'est pas certain que les projets d'équipement et d'exploitation présentés par les pétitionnaires réalisent le meilleur compromis ; à cela quatre raisons :

1) Un défaut d'imagination et de simulation qui conduit à l'équipement «rentable», sans plus, sachant que la marge économique sera simplement plus ou moins « rognée " par les exigences écologiques avancées par des services de l'Etat.

2) Quand il s'agit de renouvellement d'autorisation, on ne s'interroge pas sur l'adéquation du module d'équipement bien que deux éléments aient changé depuis le premier équipement du site :

a) l'énergie, autrefois exploitée sur place et souvent seule source d'énergie pour l'industrie qui la produisait et la consommait intégralement devait être aussi permanente que possible dans le temps; elle est maintenant la plupart du temps revendue à EDF; il peut être plus intéressant de turbiner moins longtemps, et plutôt en hiver (tarif d'achat plus intéressant) les débits importants ;

b) l'exigence d'un débit réservé substantiel réduit forcément les volumes turbinables. Il peut être intéressant d'augmenter la capacité de l'installation pour les rechercher dans des tranches de plus fort débit.

3) Un choix restreint de matériel par étroitesse du marché des gammes de turbine et le recours fréquent au marché d'occasion plutôt actif.

4) La recherche d'une rentabilité régulière moyenne d'une année à l'autre fournie par un équipement voisin du module, alors qu'un équipement supérieur serait plus spéculatif : très bon rendement les années de forte hydraulicité, très mauvais rendement les années de faible hydraulicité. Plus le débit d'équipement est élevé, plus la rentabilité est spéculative ; l'équipement au module paraît le compromis raisonnable le plus souvent utilisé.

L'objectif écologique est par nature opposé à l'objectif économique puisqu'il conduit à conserver dans le tronçon court-circuité un débit aussi naturel que possible pris sur le "stock turbinable". Il faudra donc se situer entre les deux extrêmes :

- tout le débit naturel est turbiné, tronçon court-circuité à sec en permanence ; c'est possible et ça existe !

- tout le débit naturel transite dans le lit de la rivière, il n'y a pas de débit turbiné, pas de micro centrale: c'est possible et ça existe aussi !

Entre les deux, il y a les compromis qui sont illustrés par l'étude qui suit. Les simulations se font évidemment sur des configurations réalistes intermédiaires pour montrer 
les résultats économiques et écologiques sous différentes contraintes et pour différents équipements.

\section{PROTOCOLES}

Soit un cours d'eau vosgien, la Cleurie affluent de la Moselotte ; soit une centrale hydroélectrique à Cleurie, de conception classique (prise d'eau sur seuil de $1 \mathrm{~m}$ - canal d'amenée - chambre de mise en charge - conduite forcée - usine électrique - canal de restitution) ; tronçon court-circuité de $1500 \mathrm{~m}$; la chute nette est de $3 \mathrm{~m}$.

\subsection{Paramètres de la simulation}

$Q_{\max }$ : volume maximum que peut turbiner l'installation, appelé aussi débit d'équipement.

$Q_{\text {arm }}$ : débit d'armement ; valeur plancher en-deçà duquel la turbine ne peut pas fonctionner. Il a été, dans les simulations qui suivent, fixé à $10 \%$ du $Q_{\max }$ ce qui représente la situation la plus basse connue, la plupart des turbines arment plutôt à $25-35 \%$ du $Q_{\max }$.

Ces deux paramètres sont des caractéristiques propres à une machine.

$Q_{\text {res }}$ : débit réservé fixé par la réglementation.

Chute: chute nette (= chute brute-pertes de charges singulières).

$Q_{\text {nat }}$ : débit naturel : débits moyens de chaque jour au droit du site (données fournies par le service hydrologique de la DIREN) années 84,85 et 86.

$Q_{\mathrm{dev}}$ : débit déversé (ou surversé) transitant dans le tronçon court-circuité.

Valeur du kW.h : Eté ( ${ }^{\mathrm{er}}$ avril-3l octobre) : $0,14 \mathrm{~F}$

Hiver ( $1^{\text {er }}$ novembre-31 mars) : 0,48 F.

Valeur de revente (forcée) à EDF ; d'autres tarifications spéciales existent (énergie de pointe...) qui ne sont pas envisagées ici : le kW.h hiver à $0,48 \mathrm{~F}$ comprend une prime de régularité.

\subsection{Règles de la simulation}

Le débit naturel $\left(Q_{\text {nat }}\right)$ se partage entre débit turbiné ( $Q_{\text {turb }}$ ) et débit déversé ( $\left.Q_{\mathrm{dev}}\right)$ selon la règle suivante du tableau I

\begin{tabular}{|l|c|c|}
\hline Conditions & $Q_{\mathrm{dev}}=$ & $Q_{\text {turb }}=$ \\
\hline$Q_{\text {nat }}<\left(Q_{\mathrm{res}}+Q_{\mathrm{arm}}\right)$ & $Q_{\mathrm{nat}}$ & 0 \\
$\left(Q_{\mathrm{res}}+Q_{\mathrm{arm}}\right)<\left(Q_{\mathrm{res}}+Q_{\max }\right)$ & $Q_{\mathrm{res}}$ & $Q_{\mathrm{arm}}$ à $Q_{\max }$ \\
$Q_{\mathrm{nat}}>\left(Q_{\mathrm{res}}+Q_{\max }\right)$ & $Q_{\mathrm{nat}}-Q_{\max }$ & $Q_{\max }$ \\
\hline
\end{tabular}

Ces situations sont illustrées sur les courbes de débits classés (fig. 9, 10 et II).

Une simulation est également réalisée selon laquelle, quel que soit le débit naturel, l'installation est, par disposition réglementaire, arrêtée du $1^{\mathrm{er}}$ juillet au $1^{\mathrm{er}}$ novembre - pendant cette période $Q_{\text {turb }}=0 ; Q_{\mathrm{dev}}=Q_{\text {nat. }}$.

\subsection{Calcul économique}

Revenu brut par jour: $Q_{\text {turb }} \times 24 \times 9,81 \times 0,75 \times$ chute $\times$ prix unitaire du kW.h.

Revenu brut par an: somme des revenus quotidiens pour les années 84,85 et 86 divisé par 3 .

Revenu net moyen par an: revenu brut diminué de

- $50 \mathrm{~F} / \mathrm{jour}$ de frais de maintenance et de surveillance de l'installation en service.

$-5 \% \times$ revenu brut, sur frais fixes et amortissements.

\subsection{Paramètres écologiques}

Trois paramètres sont pris en compte pour apprécier la pression que fait subir l'exploitation sur l'écosystème aquatique, salmonicole ici, dans sa partie court-circuitée.

\section{1) La valeur absolue du débit réservé}

La réduction de la lame d'eau :

— réduit l'habitabilité du milieu pour la faune piscicole par réduction de la surface en eau ou seulement de la hauteur d'eau nécessaire pour certaines espèces et/ou classes d'âge ;

- expose le milieu à l'ensoleillement qui pourra favoriser l'eutrophisation et faire déraper localement la vocation salmonicole (réchauffement);

- ne permet plus la dilution suffisante d'éventuels rejets dans le tronçon court-circuité.

\section{2) La durée d'exposition a un débit laminé}

...qui ajoute aux inconvénients visés ci-dessus celui de la durée et des effets cumulatifs aggravants. De plus, l'énergie récupérée par la centrale est non disponible dans le tronçon court-circuité pour assurer un mouvement normal des sédiments et le décolmatage du lit.

\section{3) La période où apparaît la situation de laminage}

Là encore, ce paramètre intervient en potentialisant les effets cités au 1); on citera notamment le laminage des débits d'été qui peuvent aggraver une situation «naturelle " déjà tendue du point de vue de la qualité ; c'est à ce titre qu'une simulation est réalisée en arrêtant l'exploitation du $1^{\text {er }}$ juillet au $1^{\text {er }}$ novembre.

Il intervient en outre pendant la période où les truites remontent sur les frayères (sept., oct. dans les Vosges) : à l'aval de la restitution, si le débit turbiné est supérieur au débit déversé, il y a toutes les chances pour que les truites remontent dans le canal de fuite (qui représente le courant principal donc plus attractif) où elles resteront bloquées à l'aval des turbines, au lieu de s'engager dans le lit naturel pour remonter jusqu'au barrage de prise d'eau où se situe en général l'ouvrage de franchissement. 
Tableau 1. - Evolution des principaux paramètres résultant de différentes combinaisons de capacité installée $\left(Q_{\max }\right)$, de débits réservés $\left(Q_{\text {res }}\right)$ d'arrêt estival ou non.

\begin{tabular}{|l|c|c|c|c|c|c|}
\hline & \multicolumn{3}{|c|}{ sans arrêt estival } & \multicolumn{3}{c|}{ arrêt estival } \\
\hline$Q_{\text {arm }}=$ & 0,2 & 0,3 & 0,5 & 0,2 & 0,3 & 0,5 \\
$Q_{\max }=$ & 2 & 3 & 5 & 2 & 3 & 5 \\
$Q_{\text {res }}=$ & 0,2 & 0,2 & 0,2 & 0,2 & 0,2 & 0,2 \\
vol. turbiné $\%=$ & 55 & 66 & 75 & 43 & 53 & 63 \\
REVBRUT/AN $=$ & 76117 & 92677 & 109313 & 68451 & 84034 & 101241 \\
REVNET/AN $=$ & 53995 & 70027 & 89364 & 52862 & 67699 & 84679 \\
nb. j. où $Q_{\text {turb }}=0$ & 0 & 5 & 76 & 123 & 123 & 136 \\
nb. j. où $Q_{\text {dev }}=Q_{\text {res }}$ & 232 & 286 & 254 & 129 & 179 & 198 \\
nb. j. où $Q_{\text {turb }}=Q_{\text {max }}$ & 134 & 74 & 36 & 115 & 64 & 32 \\
nb. j. où $Q_{\text {turb }}=Q_{\text {dev }}$ & 310 & 334 & 281 & 195 & 219 & 223 \\
\hline
\end{tabular}

\begin{tabular}{|l|c|c|c|c|c|c|}
\hline$Q_{\text {arm }}=$ & 0,2 & 0,3 & 0,5 & 0,2 & 0,3 & 0,5 \\
$Q_{\max }=$ & 2 & 3 & 5 & 2 & 3 & 5 \\
$Q_{\text {res }}=$ & 0,3 & 0,3 & 0,3 & 0,3 & 0,3 & 0,3 \\
vol. turbiné $\%=$ & 52 & 62 & 72 & 42 & 51 & 60 \\
REVBRUT/AN $=$ & 72731 & 87389 & 104579 & 65914 & 80345 & 97201 \\
REVNET/AN $=$ & 51044 & 67436 & 85517 & 50452 & 64494 & 81074 \\
nb. j. où $Q_{\text {turb }}=0$ & 5 & 54 & 89 & 123 & 129 & 140 \\
nb. j. où $Q_{\text {dev }}=Q_{\text {res }}$ & 235 & 241 & 243 & 136 & 176 & 196 \\
nb. j. où $Q_{\text {turb }}=Q_{\max }$ & 126 & 70 & 34 & 107 & 60 & 29 \\
nb. j. où $Q_{\text {turb }}=Q_{\text {dev }}$ & 256 & 285 & 268 & 189 & 213 & 218 \\
\hline
\end{tabular}

\begin{tabular}{|l|c|c|c|c|c|c|}
\hline$Q_{\text {arm }}=$ & 0,2 & 0,3 & 0,5 & 0,2 & 0,3 & 0,5 \\
$Q_{\max }=$ & 2 & 3 & 5 & 2 & 3 & 5 \\
$Q_{\text {res }}=$ & 0,4 & 0,4 & 0,4 & 0,4 & 0,4 & 0,4 \\
vol. turbiné $\%=$ & 49 & 58 & 68 & 40 & 49 & 58 \\
REVBRUT/AN $=$ & 68628 & 82997 & 100106 & 63029 & 76714 & 93216 \\
REVNET/AN $=$ & 49614 & 64381 & 81718 & 48028 & 61395 & 77539 \\
nb. j. où $Q_{\text {turb }}=0$ & 54 & 76 & 98 & 129 & 136 & 145 \\
nb. j. où $Q_{\text {dev }}=Q_{\text {res }}$ & 193 & 221 & 236 & 136 & 171 & 193 \\
nb. j. où $Q_{\text {turb }}=Q_{\text {max }}$ & 119 & 68 & 32 & 101 & 58 & 27 \\
nb. j. où $Q_{\text {turb }}=Q_{\text {dev }}$ & 221 & 250 & 259 & 177 & 202 & 213 \\
\hline
\end{tabular}

\begin{tabular}{|l|c|c|c|c|c|c|}
\hline$Q_{\text {arm }}=$ & 0,2 & 0,3 & 0,5 & 0,2 & 0,3 & 0,5 \\
$Q_{\max }=$ & 2 & 3 & 5 & 2 & 3 & 5 \\
$Q_{\text {res }}$ & 0,5 & 0,5 & 0,5 & 0,5 & 0,5 & 0,5 \\
vol. turbiné $\%=$ & 46 & 55 & 65 & 38 & 47 & 55 \\
REVBRUT/AN $=$ & 65157 & 79115 & 95428 & 60149 & 73363 & 89017 \\
REVNET/AN $=$ & 47366 & 61293 & 77856 & 45609 & 58395 & 73900 \\
nb. j. où $Q_{\text {turb }}=0$ & 76 & 89 & 111 & 136 & 140 & 153 \\
nb. j. où $Q_{\text {dev }}=Q_{\text {res }}$ & 180 & 211 & 225 & 137 & 169 & 187 \\
nb. j. où $Q_{\text {turb }}=Q_{\text {max }}$ & 110 & 67 & 31 & 94 & 57 & 27 \\
nb. j. où $Q_{\text {turb }}=Q_{\text {dev }}$ & 199 & 229 & 246 & 164 & 189 & 205 \\
\hline
\end{tabular}

\begin{tabular}{|l|c|c|c|c|c|c|}
\hline$Q_{\text {arm }}=$ & 0,2 & 0,3 & 0,5 & 0,2 & 0,3 & 0,5 \\
$Q_{\max }=$ & 2 & 3 & 5 & 2 & 3 & 5 \\
$Q_{\text {res }}=$ & 0,6 & 0,6 & 0,6 & 0,6 & 0,6 & 0,6 \\
vol. turbiné $\%=$ & 44 & 53 & 62 & 37 & 44 & 53 \\
REVBRUT/AN $=$ & 62006 & 75445 & 90698 & 57417 & 70087 & 84726 \\
REVNET/AN $=$ & 44972 & 58239 & 74097 & 43179 & 55516 & 70340 \\
nb. j. où $Q_{\text {turb }}=0$ & 89 & 98 & 125 & 140 & 145 & 164 \\
nb. j. où $Q_{\text {dev }}=Q_{\text {res }}$ & 175 & 205 & 212 & 140 & 166 & 177 \\
nb. j. où $Q_{\text {turb }}=Q_{\text {max }}$ & 104 & 64 & 30 & 88 & 56 & 26 \\
nb. j. où $Q_{\text {turb }}=Q_{\text {dev }}$ & 174 & 204 & 221 & 146 & 171 & 187 \\
\hline
\end{tabular}


Ces paramètres sont lisibles sur les hydrogrammes, ils sont exprimés également dans le tableau 1 par les paramètres «nombre de jours par an où "

$Q_{\text {turb }}=0$ : le tronçon court-circuité transite l'ensemble du débit naturel.

$Q_{\mathrm{dev}}=Q_{\mathrm{res}}$ : le débit déversé est uniformément celui du débit réservé.

$Q_{\text {turb }}=Q_{\max }$ : le débit déversé varie comme le débit naturel; il lui est simplement inférieur de la valeur $Q_{\max }$.

$Q_{\text {turb }}>Q_{\text {dev }}$ : le débit turbiné attire les truites dans l'impasse du canal de fuite.

\section{III $\square$ RÉSULTATS (tabl. 2)}

La séquence des débits utilisée concerne les années 84,85 , 86; la Cleurie a écoulé pendant cette période $225606384 \mathrm{~m}^{3}$.

Avec des modules annuels respectivement de 2,49, 1,84 et $2,81 \mathrm{~m}^{3} / \mathrm{s}$, cette période a un débit moyen de $2,38 \mathrm{~m}^{3} / \mathrm{s}$, comparable au module de référence moyen interannuel $\left(2,32 \mathrm{~m}^{3} / \mathrm{s}\right)$. Les années 85 et 86 ont des débits contrastés, significatifs d'année sèche (85) et d'année humide (86).

Les simulations ont été faites en croisant trois paramètres offrant chacun et respectivement 3,5 et 2 modalités, conduisant à 30 combinaisons analysées ici.
$Q_{\max }: 2 \mathrm{~m}^{3} / \mathrm{s}, 3 \mathrm{~m}^{3} / \mathrm{s}$ et $5 \mathrm{~m}^{3} / \mathrm{s}$ soit $0,86,1,29$ et 2,16 fois le module interannuel.

Le débit d'armement ( $Q_{\text {arm }}$ ) représente toujours $10 \%$ du débit maximum. Il est sans doute un peu sous-évalué.

$Q_{\text {res }}$ : débit réservé

5 modalités : 0,$2 ; 0,3 ; 0,4 ; 0,5 ; 0,6 \mathrm{~m}^{3} / \mathrm{s}$ soit $9,13,17$, $22,26 \%$ du module interannuel.

Arrêt estival : modalités : sans arrêt ;

arrêt forcé du $1^{\text {er }}$ juillet au $1^{\text {er }}$ novembre.

3.1 Effet de la configuration sur le revenu ( $f i g$ l)

Le revenu croît régulièrement avec la capacité installée, en simulation arrêt estival ou non; ceci s'explique par une plage de travail plus vaste quand on augmente la capacité et une meilleure exploitation de l'énergie l'hiver.

Certes une extrapolation au-delà de cette fourchette aurait bien fini par donner un point d'inflexion ; il convient cependant d'apprécier que le surdimensionnement sera soumis à des contraintes techniques et financières peu réalistes.

Le revenu net baisse linéairement quand le débit réservé augmente. Logique du " manque à turbiner »; la décroissance est plus forte en valeur absolue pour un équipement à $5 \mathrm{~m}^{3} / \mathrm{s}(-15267 \mathrm{~F})$ qu'à $2 \mathrm{~m}^{3} / \mathrm{s}(-9023 \mathrm{~F})$, mais moindre en valeur relative (respectivement 13 et $17 \%$ ).

Tableau 2. - Évolution des revenus (R) et temps de retour d'investissement (TR) pour différentes combinaisons de débit réservé $x$ équipement.

\begin{tabular}{|c|c|c|c|}
\hline & $2 \mathrm{~m}^{2} / \mathrm{s}$ & $3 \mathrm{~m}^{2} / \mathrm{s}$ & $5 \mathrm{~m}^{2} / \mathrm{s}$ \\
\hline$Q_{\mathrm{res}}=0,2$ & $\begin{array}{c}\mathrm{R}=53995 \mathrm{~F} \\
\text { (référence) } \\
\mathrm{TR}=9,7 \text { années }\end{array}$ & $\begin{array}{c}\mathrm{R}=70027 \mathrm{~F} \\
\text { (référence) } \\
\mathrm{TR}=9,8 \text { années }\end{array}$ & $\begin{array}{c}\mathrm{R}=89364 \mathrm{~F} \\
\text { (référence) } \\
\mathrm{TR}=11,0 \text { années }\end{array}$ \\
\hline$Q_{\mathrm{res}}=0,3$ & $\begin{array}{c}R=51044 \mathrm{~F} \\
P=-4,5 \% \\
T R=10,3 \text { années }\end{array}$ & $\begin{array}{c}\mathrm{R}=67436 \mathrm{~F} \\
\mathrm{P}=-3,7 \% \\
\mathrm{TR}=10,2 \text { années }\end{array}$ & $\begin{array}{c}\mathrm{R}=85517 \mathrm{~F} \\
\mathrm{P}=-4,3 \% \\
\mathrm{TR}=11,5 \text { années }\end{array}$ \\
\hline$Q_{\mathrm{res}}=0,4$ & $\begin{array}{c}\mathrm{R}=49614 \mathrm{~F} \\
\mathrm{P}=-8 \% \\
\mathrm{TR}=10,6 \text { années }\end{array}$ & $\begin{array}{c}R=64381 \mathrm{~F} \\
P=-8,1 \% \\
T R=10,7 \text { années }\end{array}$ & $\begin{array}{c}\mathrm{R}=81718 \mathrm{~F} \\
\mathrm{P}=-8,6 \% \\
\mathrm{TR}=12,1 \text { années }\end{array}$ \\
\hline$Q_{\mathrm{res}}=0,5$ & $\begin{array}{c}\mathrm{R}=47366 \mathrm{~F} \\
\mathrm{P}=-12 \% \\
\mathrm{TR}=11,1 \text { années }\end{array}$ & $\begin{array}{c}R=61293 \mathrm{~F} \\
P=-12,5 \% \\
T R=11,3 \text { années }\end{array}$ & $\begin{array}{c}\mathrm{R}=77856 \mathrm{~F} \\
\mathrm{P}=-13 \% \\
\mathrm{TR}=12,7 \text { années }\end{array}$ \\
\hline$Q_{\mathrm{res}}=0,6$ & $\begin{array}{c}R=44972 F \\
P=-16 \% \\
T R=11,4 \text { années }\end{array}$ & $\begin{array}{c}\mathrm{R}=58239 \mathrm{~F} \\
\mathrm{P}=-17 \% \\
\mathrm{TR}=11,8 \text { années }\end{array}$ & $\begin{array}{c}R=74097 \mathrm{~F} \\
P=-17 \% \\
T R=13,3 \text { années }\end{array}$ \\
\hline
\end{tabular}

R : Recettes, P : Pourcentage de perte, TR: Temps de retour.

\begin{tabular}{|l|c|c|c|}
\hline Puissance nette & $44,1 \mathrm{~kW}$ & $66,2 \mathrm{~kW}$ & $110 \mathrm{~kW}$ \\
\hline Coût du kW installé & 12000 & 10500 & 9000 \\
\hline $\begin{array}{l}\text { Investissement } \\
\text { moyen }\end{array}$ & 528000 & 693000 & 990000 \\
\hline
\end{tabular}



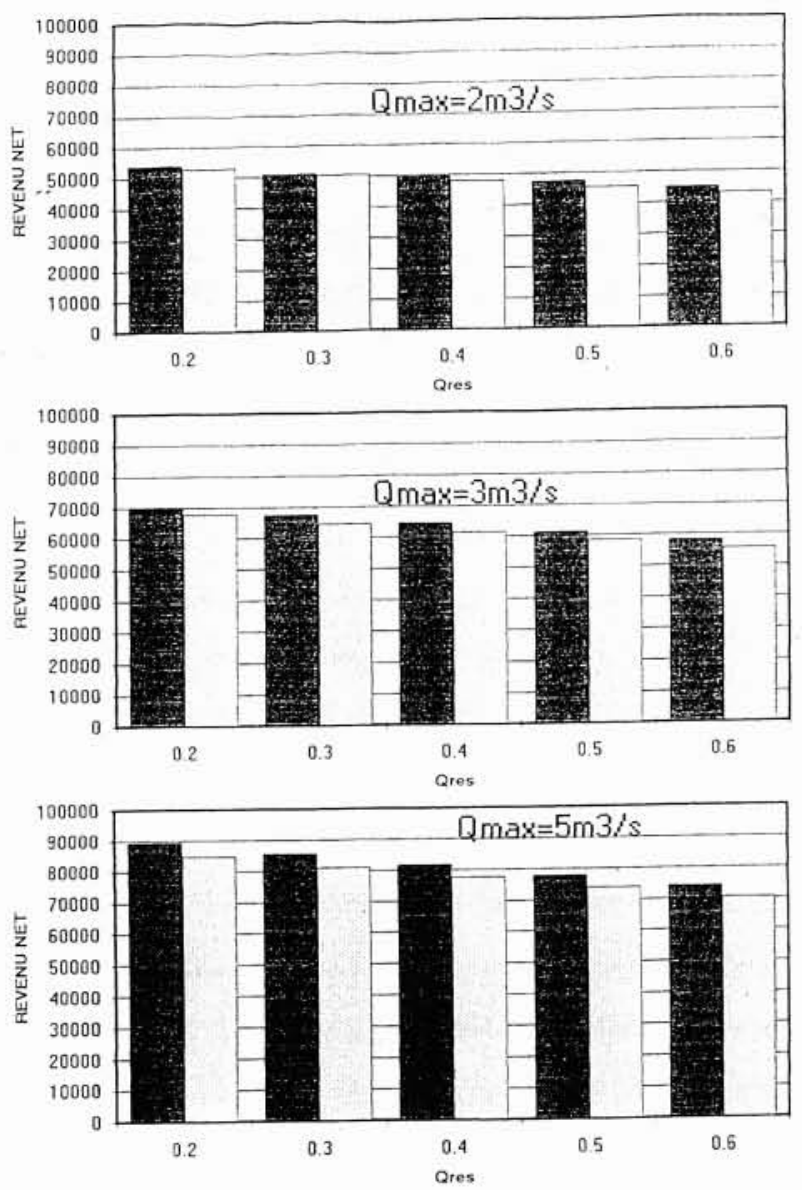

1. Evolution du revenu net/an pour la période 84 à 86 en fonction du débit d'équipement et du débit réservé. Les colonnes foncées représentent l'exploitation sans contrainte estivale et les colonnes claires, avec un arrêt forcé du $1^{\text {er }}$ juillet au $1^{\text {er }}$ novembre.

L'arrêt du turbinage d'été réduit peu significativement les revenus et dans un rapport qui paraît linéaire et constant.

Le revenu augmente avec le suréquipement et il est moins érodé en valeur relative par un débit réservé plus élevé ; par exemple l'option la plus exigeante écologiquement ( $Q_{\text {res }}=0,6 \mathrm{~m}^{3} / \mathrm{s}$, arrêt d'été) pour un équipement à $3 \mathrm{~m}^{3} / \mathrm{s}$ offre un revenu (55 516 F/an) supérieur à l'option la plus laxiste écologiquement ( $Q_{\mathrm{res}}=0,2 \mathrm{~m}^{3} / \mathrm{s}$, pas d'arrêt estival) pour un équipement de $2 \mathrm{~m}^{3} / \mathrm{s}(53995 \mathrm{~F})$.

En conclusion et dans la plage de simulation, le suréquipement à revenu équivalent apporte une meilleure préservation du milieu naturel.

Le tableau 3 rend compte de l'augmentation linéaire des coûts en fonction du suréquipement et de l'augmentation du débit réservé ; mais on voit par ailleurs que les rendements « écologiques » corrélés sont plutôt exponentiels.

\subsection{Effet de la configuration sur le partage des débits}

Les figures 2, 3 et 4 confirment l'observation qui précède ; dans la comparaison citée plus haut, l'exigence d'un arrêt
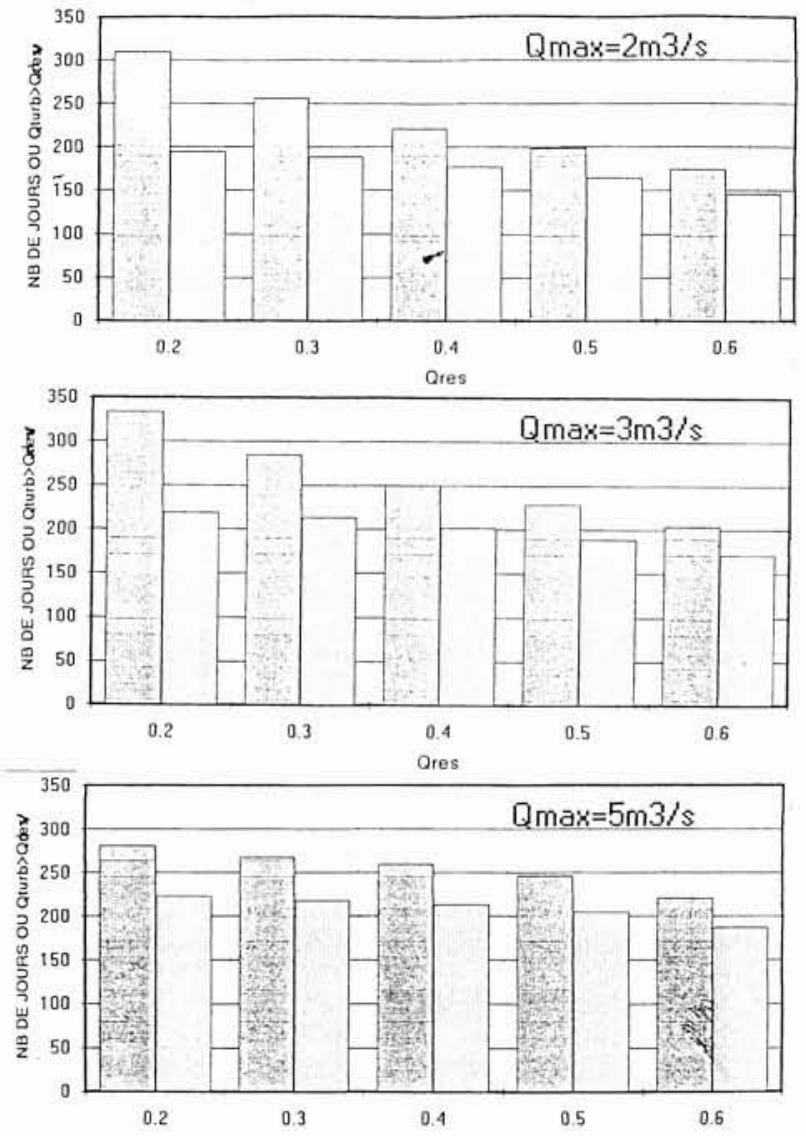

2. Evolution du nombre de jours où le débit turbiné est supérieur au débit déversé, en fonction du débit d'équipement $Q_{\max }$ et du débit réservé $Q_{\mathrm{res}}$ (colonnes foncées : exploitation sans contrainte estivale, colonnes claires : arrêt forcé $1^{\text {er }}$ juillet-1 ${ }^{\text {er }}$ novembre).

du turbinage en été est sensible pour les débits réservés les plus faibles, moins pour les débits réservés les plus élevés. A économie comparable, le débit déversé est réduit au débit réservé pendant $232 \mathrm{j} / \mathrm{an}\left(Q_{\max }=2 \mathrm{~m}^{3} / \mathrm{s} ; Q_{\text {res }}=\right.$ $0,2 \mathrm{~m}^{3} / \mathrm{s}$ ) alors que cette situation n'est rencontrée que 169 jours/an quand $Q_{\max }=3 \mathrm{~m}^{3} / \mathrm{s}$ et $Q_{\text {res }}=0,6 \mathrm{~m}^{3} / \mathrm{s}$.

On comparera utilement les situations où le débit naturel transite intégralement dans le tronçon court-circuité, situation on ne peut plus écologique.

\section{Conclusion :}

Un équipement significativement supérieur en module permet d'augmenter sensiblement les revenus tout en durcissant les règles de préservation des débits déversés.

La recommandation naturelle qui en découle se heurte à la vérification que le suréquipement, assorti de contraintes écologiques fortes ou non, conduit à une plus forte disparité des revenus selon que l'année est sèche ou humide. Dès lors, les promoteurs qui empruntent l'essentiel du budget d'investissement auprès des banques rassurées par le revenu issu d'un marché captif (revente à EDF) préféreront un revenu (pour des remboursements) régulier plutôt que d'assurer l'inertie financière compensant les 

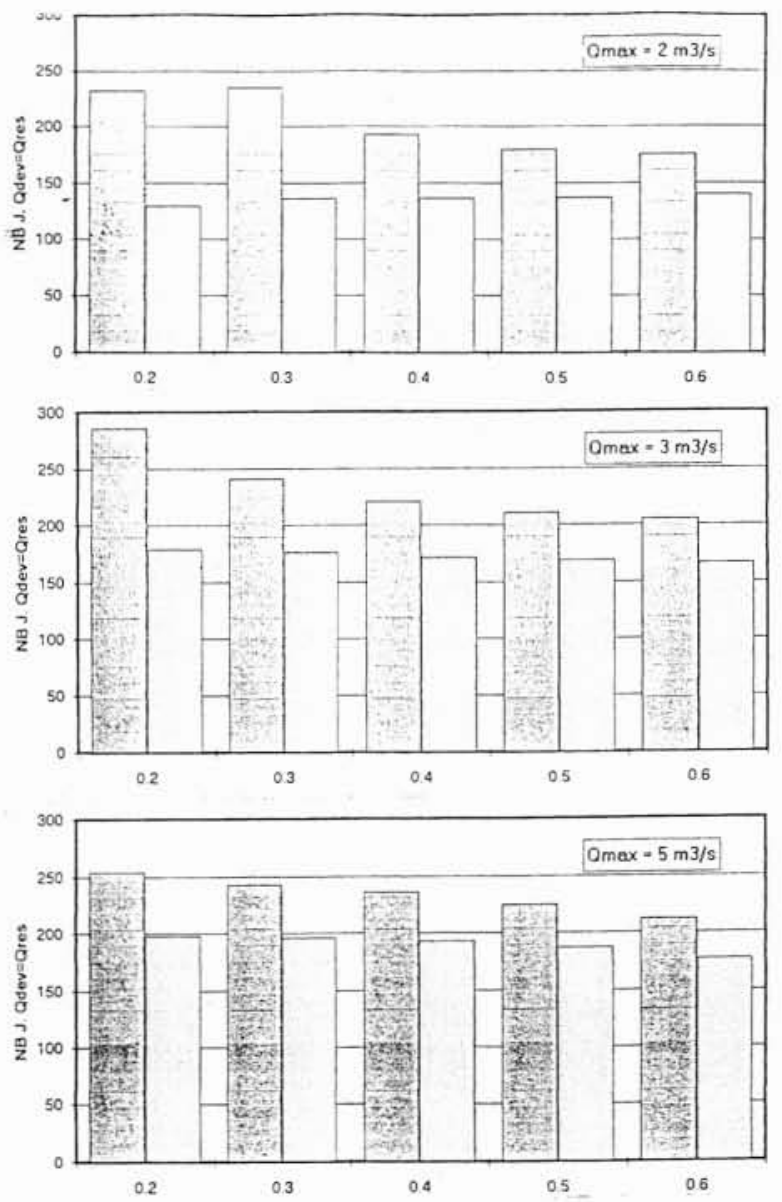

3. Evolution du nombre de jours où le débit déversé est réduit au seul débit réservé, en fonction du débit d'équipement $Q_{\max }$ et du débit réservé $Q_{\text {res }}$ (colonnes foncées : exploitation sans contrainte estivale ; colonnes claires : arrêt forcé $1^{\text {er }}$ juillet-1 ${ }^{\text {er }}$ novembre).

aléas liés à la variabilité des débits naturels. L’absence de risque économique conduit donc à une rente basée sur le risque écologique.

\subsection{Simulations sur hydrogrammes}

Les simulations qui précèdent, illustrent seulement la démarche qu'on est en droit d'attendre de n'importe quel promoteur sollicitant une autorisation ou concession, ou le renouvellement de celles-ci. N'importe quelle étude ou notice d'impact doit fournir les variantes (ici les différentes combinaisons) et les raisons du choix. C'est à la fois pour le promoteur un moyen d'optimiser son choix économique, pour l'administration garante de la conservation du patrimoine aquatique et de la pertinance industrielle du projet, un moyen de choix d'une variante qui dans tous les cas est un compromis entre économie et écologie le plus équitable possible.

Les simulations sur les hydrogrammes permettent de visualiser sur des années-types le partage du débit turbiné
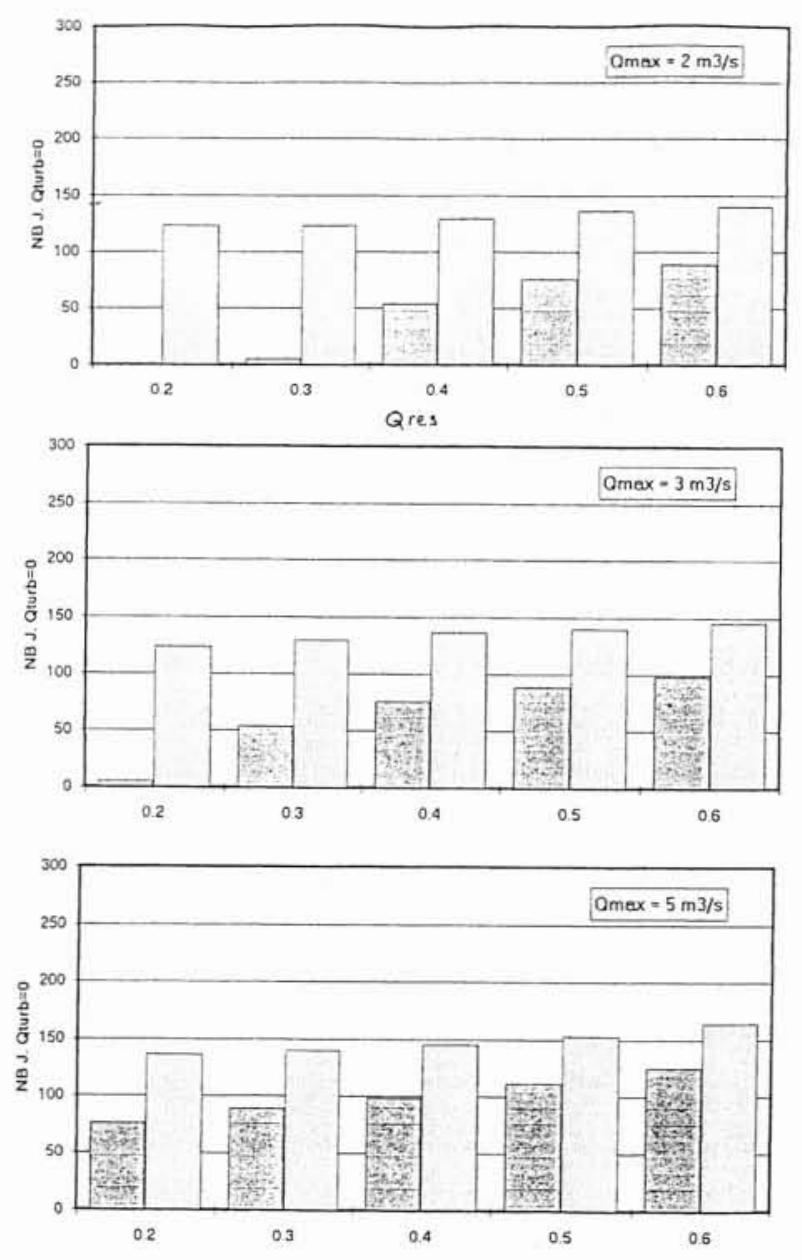

4. Evolution du nombre de jours (an où l'installation ne fonctionne pas $\left(Q_{\mathrm{dev}}=Q_{\mathrm{nat}}\right)$ en fonction du débit d'équipement $Q_{\max }$ et du débit réservé $Q_{\text {res }}$ (colonnes foncées : exploitation sans contrainte estivale ; colonnes claires : arrêt forcé $1^{\text {er }}$ juillet-1 ${ }^{\text {er }}$ novembre).

et surversé en fonction des contraintes. Les combinaisons sont celles qui ont fait l'objet de bilan économiqueécologique.

Sur la figure 5, pour 3 années type et pour une combinaison donnée, on observe :

a) que les années se suivent et ne se ressemblent pas (mais allez expliquer ça à un banquier !),

b) que l'arrêt du turbinage en été a permis aux truites d'exploiter au mieux pour leur migration les «coups d'eau " de septembre, octobre,

c) que ce même arrêt des turbines en 1985 (année sèche) a évité d'aggraver une situation d'étiage tendue en préservant dans la partie naturelle l'intégralité du débit.

Les figures 6, 7 et 8 illustrent les combinaisons expertisées pour l'année 1984.

Sur la figure $6 a$, on voit que le turbinage ne s'arrête jamais, qu'il laisse dans le tronçon court-circuité un débit laminé pendant la période la plus sensible de l'été que les coups d'eau de l'automne vont se partager entre les deux voies $Q_{\text {turb }}$ et $Q_{\mathrm{dev}}$ où les truites vont s'égarer. 

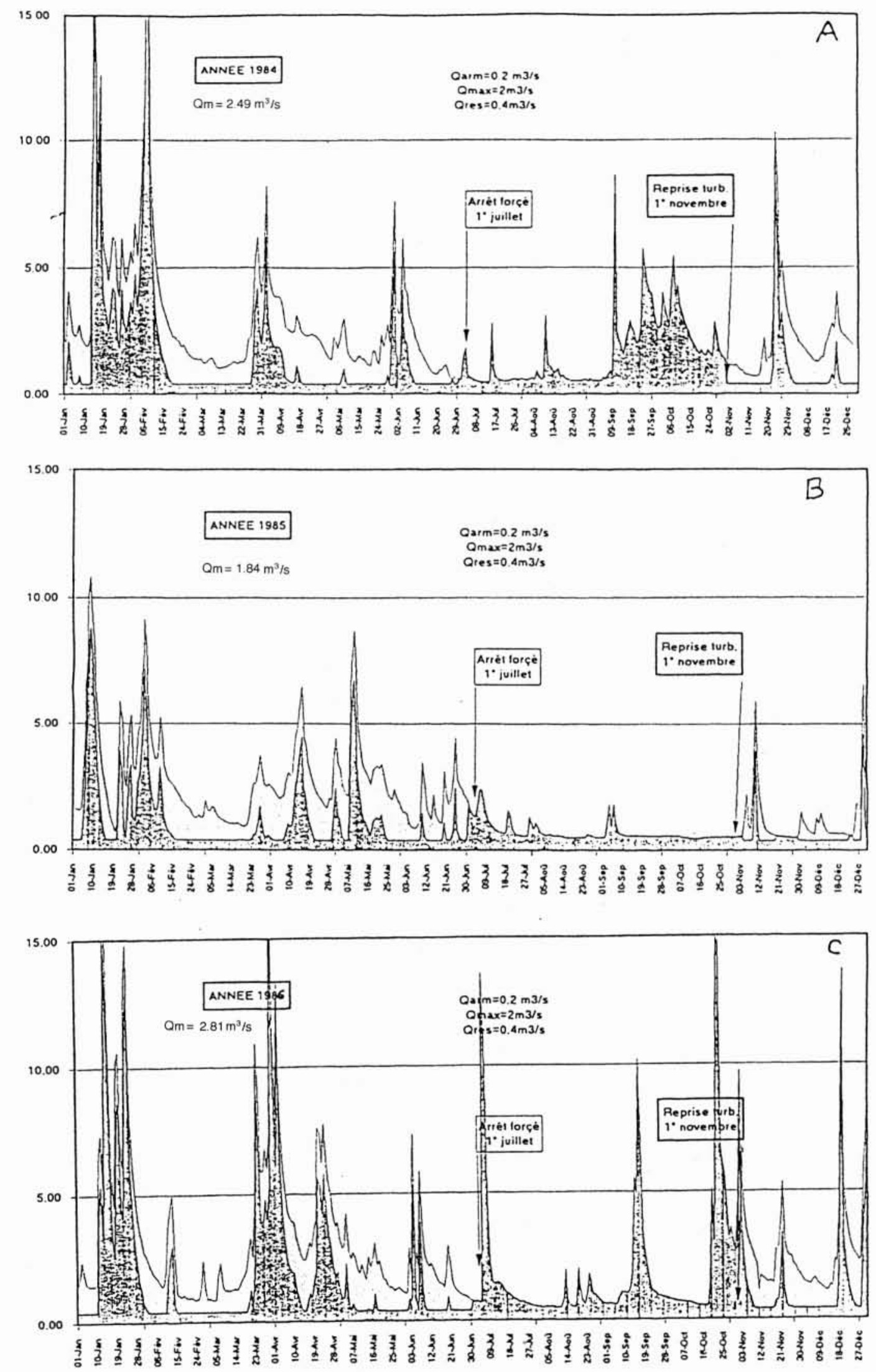

5. Hydrogrammes des années 84,85 et 86 de la Cleurie à Cleurie (88), partage du débit naturel (foncées) et prise d'eau pour alimenter la turbine (claires). 


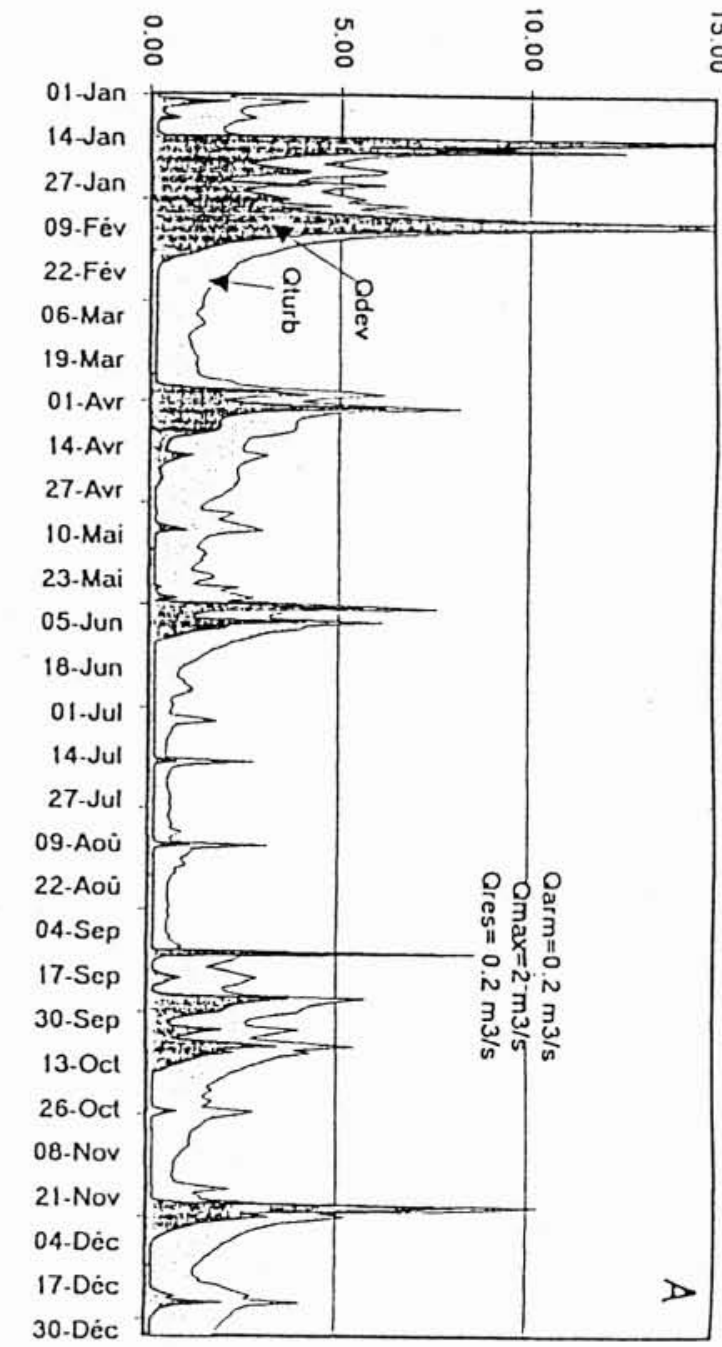



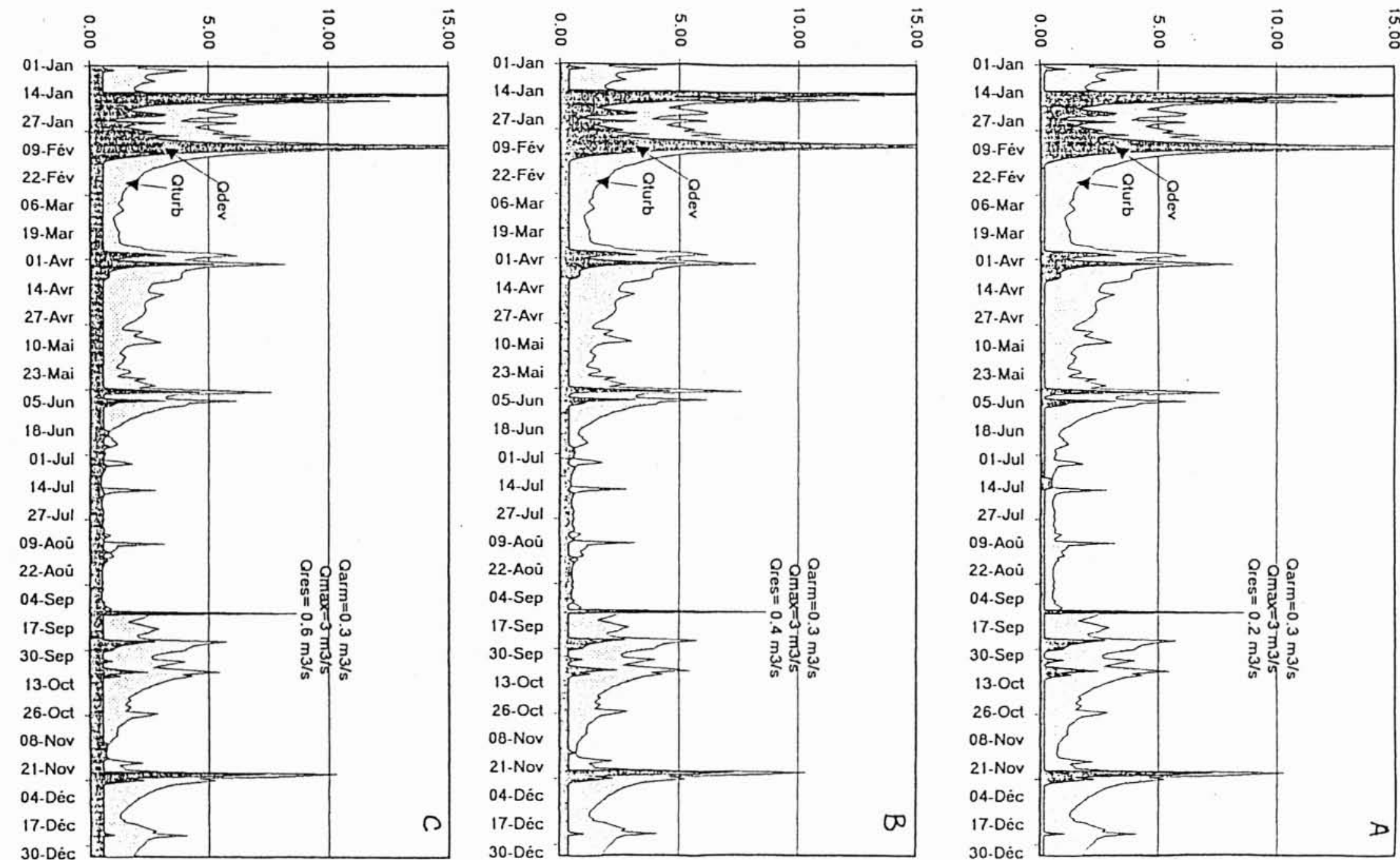

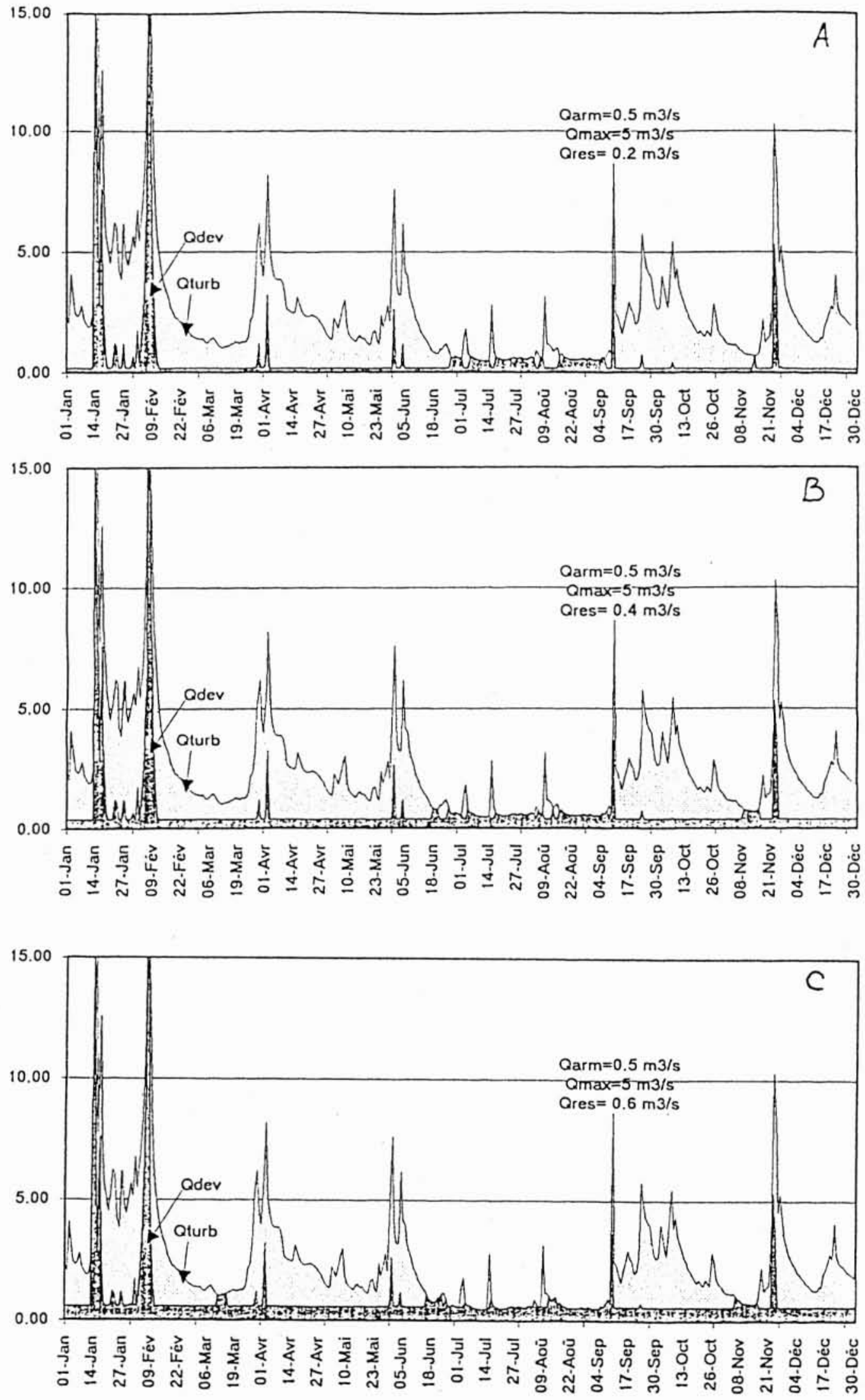

8. Simulation du partage des débits pour l'année 1984. Débit d'équipement $=5 \mathrm{~m}^{3} / \mathrm{s} ; Q_{\mathrm{res}}=0,2 \mathrm{~m}^{3} / \mathrm{s}(\mathrm{A}) ; 0,4 \mathrm{~m}^{3} / \mathrm{s}(\mathrm{B})$; $0,6 \mathrm{~m}^{3} / \mathrm{s}(\mathrm{C})$. 
On voit sur les figures suivantes 7 et 8 que les volumes exploités notamment en hiver (donc les bénéfices) sont meilleurs sur la figure 8-A; on observe le laminage du débit déversé au printemps et début d'été ; les eaux sont encore fraîches et les alevins petits. D'aucuns auraient vérifié l'effet bénéfique de ce «lissage» des crues de printemps sur la survie des alevins et truitelles; bénéfice économique et bénéfice écologique ; à quoi bon "grignoter » les débits d'été à $0,14 \mathrm{~F} / \mathrm{kW} \cdot \mathrm{h}$ !

\section{IV $\square$ CONCLUSION}

Ce travail illustre une réflexion et une démarche sans prétendre à l'exhaustivité. La facilité de mise en œuvre par des moyens grand public de la micro informatique plaide pour une exploration plus complète des variantes possibles. Autour de celle qui sert de prétexte ici, il peut en exister de nombreuses telles que la modulation du débit réservé en fonction de la saison, en fonction de paramètres hydrologiques ou hydrobiologiques instantanés.

Le propositions, qui relèvent clairement de la compétence et de l'obligation faites au maître d'ouvrage pour
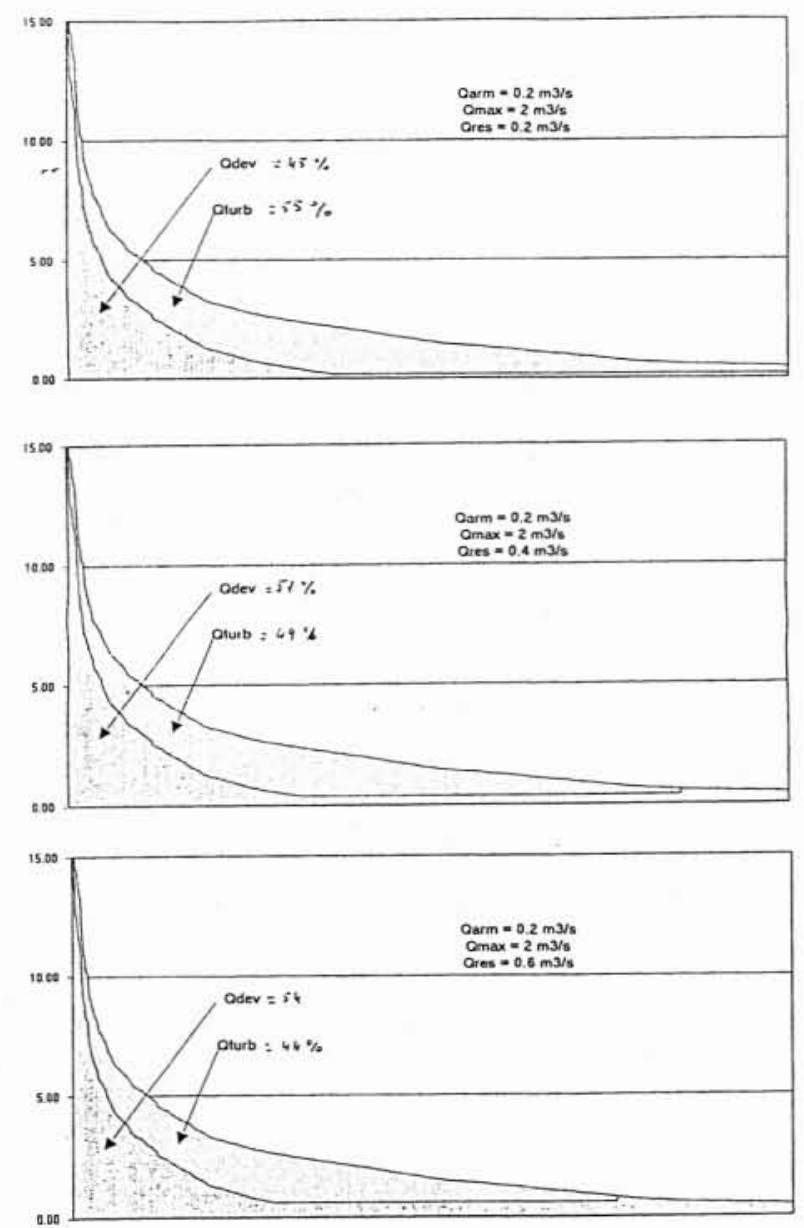

9. Courbe des débits classés des 3 années $84,85,86$; partage des débits en fonction du débit réservé imposé. (Débit d'équipement $=2 \mathrm{~m}^{3} / \mathrm{s}$.) éclairer la décision administrative, pourront lui être profitable en retour : partenaire objectif de l'administration de l'Etat dans un choix d'équipement et d'exploitation, il pourra plus facilement adapter son mode de gestion et règlement d'eau à d'éventuelles contraintes futures comme des exigences écologiques accrues ou la révision toujours possible des contrats de vente auprès d'EDF (harmonisation européenne des tarifs d'énergie renouvelable, abandon de l'obligation de rachat).

La légitimité d'exploitation d'un droit d'eau n'est pas remise en cause ; mais dans le contexte économique protégé par un écoulement automatique et non spéculatif du produit, on est en droit d'attendre de cette industrie qu'elle propose :

a) une optimisation économique d'installation et fonctionnement,

b) une expression claire, et justifiée de ses projets,

c) une prise de risque économique qui lui appartient et qu'elle peut partager dans le cadre de produit financier existant et adapté plutôt que la prise du risque écologique qui ne lui appartient pas.
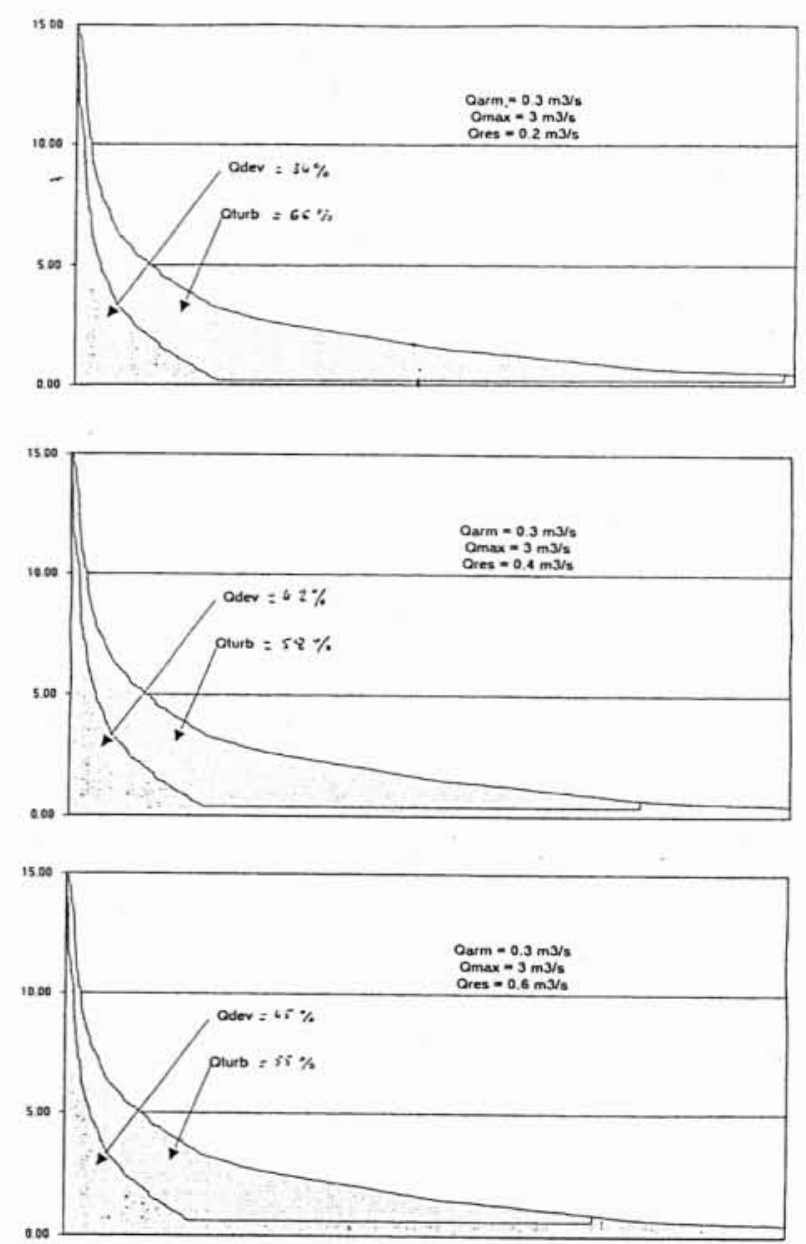

10. Courbe des débits classés des 3 années $84,85,86$; partage des débits en fonction du débit réservé imposé. (Débit d'équipement $=3 \mathrm{~m}^{3} / \mathrm{s}$ ). 

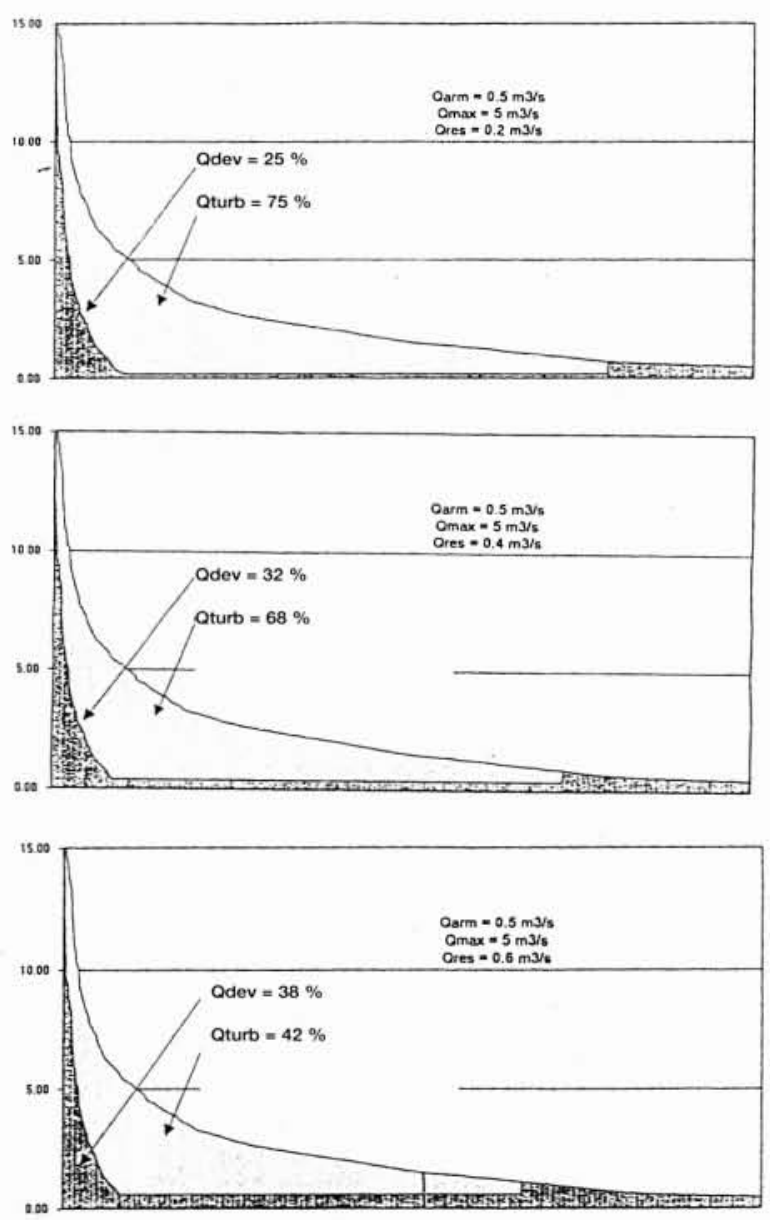

11. Courbe des débits classés des 3 années $84,85,86$; partage des débits en fonction du débit réservé imposé. (Débit d'équipement $=5 \mathrm{~m}^{3} / \mathrm{s}$ ).

\section{Bibliographie}

[1] A.F.M.E. (1985). - Petite hydraulique. Problématique de développement. Compte-rendu du colloque Valbonne, Sophia Antipolis, $256 \mathrm{p}$.

[2] Bietz B., MARTIN J. (1985), - Exigences de débit du courant pour les poissons nageant en aval des aménagements hydroélectriques. Rapport destiné à l'association canadienne d'électricité, Montréal (Québec), 87 p.

[3] DELARUE J. (1992). - Les microcentrales hydroélectriques. C.R. de l'assemblée générale du Club Halieutique Interdépart. Perpignan, $7 \mathrm{p}$.

[4] Do T. (1993). - La détermination du débit réservé impose aux ouvrages hydrauliques en général et aux petites centrales hydrauliques en particulier, l'expérience Lorraine. Doc Diren Lorraine-SEMA, 9 p. + annexes.

[5] EDF DROz S. (197?). - Les microcentrales hydroélectriques, Principe et pratique. Information et Communication EDF dir. de l'Equipement cote: W13, 35 p.

[6] Electricité autonome française, hydro-M (1989). — Débits réservés et aménagements hydroélectriques. Hydro-M. Toulouse, $179 \mathrm{p}+$ annexes.

[7] FREIRE FORMIGA R.M. - Les conséquences des débits réserves réglementaires. Analyse des cas d'ouvrages hydroélectriques. D.E.A. univ. Paris XII, ENPC, ENGREF, 50 p. + annexes.

[8] Ginocchio R. - L'énergie hydraulique. Eyrolles Ed.

[9] J.O.R.F. 1981 : Décret $N^{\circ} 81-375$ du 15 avril 1981. Modifiant l'article 16 de la loi du 16 octobre 1919 modifiée relative à l'utilisation de l'énergie hydroélectrique et pris pour son application en ce qui concerne la forme et la procédure d'instruction des demandes d'autorisation d'usines hydroélectriques. J.O. du 19 avril 1981.

[10] SABAton C. (1991). - Quel débit réserver à l'aval des ouvrages hydroélectriques? La méthode des microhabitats : un outil d'aide à la décision. Note technique E.D.F., D.E.R., ref. HE31/91-26, $62 \mathrm{p}$. 